\title{
The investigation of four technologies to assist in detecting mild to moderate traumatic brain injury of U.S. Military service members
}

\author{
Valerie Rice $^{\mathrm{a}, *}$, Gary Boykin ${ }^{\mathrm{a}}$, Petra Alfred ${ }^{\mathrm{a}}$, Gaylord Lindsay ${ }^{\mathrm{b}}$, Cory Overby $^{\mathrm{b}}$, Angela Jeter ${ }^{\mathrm{b}}$, \\ Mark Lester ${ }^{c}$, Stephen Allison ${ }^{\mathrm{d}}$ and Douglas Cooper ${ }^{\mathrm{e}}$ \\ ${ }^{a}$ U.S. Army Research Laboratory, JBSA Fort Sam Houston, TX, USA \\ ${ }^{\mathrm{b}}$ General Dynamics Corporation, Falls Church, VA, USA \\ ${ }^{\mathrm{c}}$ Brooke Army Medical Center, Institute for Surgical Research Laboratory, JBSA Fort Sam Houston, TX, USA \\ ${ }^{\mathrm{d}}$ U.S. Army Research Institute of Environmental Medicine, Natick, MA, USA \\ ${ }^{\mathrm{e}}$ San Antonio Area Medical Center, JBSA Fort Sam Houston, TX, USA
}

Received 13 March 2018

Accepted 8 August 2018

\begin{abstract}
. traumatic brain injury (TBI). TBI. for a negative result with zero measures being positive. presence of mild to moderate TBI.

*Address for correspondence: Valerie J. Rice, U.S. Army Research Laboratory Army Medical Department Field Element, 3161 McIndoe Rd., Building 1152, Suite \#1, Fort Sam Houston (JBSA), San Antonio, TX, 78234 7731, USA. Tel.: +1 210221

2007; E-mail: Valerie.j.rice.civ@mail.mil.
\end{abstract}

BACKGROUND: A standard, reliable, objective measure is needed for identifying individuals with mild to moderate

OBJECTIVE: The purpose of this study was to examine balance using an AMTI OR6-7 force platform (FP), neurocognition and mood using the Automated Neuropsychological Assessment Metric4 (ANAM4), blood flow comparisons using a Brain Acoustic Monitor (BAM), and voice using Voice Analysis software (VA) for screening service members for a mild to moderate

METHODS: Active duty and retired service member volunteers ( $n=88,35$ with a diagnosis of mild to moderate TBI and 53 who never had a TBI) completed an informed consent document, and evaluations using the four technologies.

RESULTS: Development of a clinical prediction rule yielded two FP variables and one ANAM4 Mood Scale variable (vigor) as helpful in predicting the presence of a TBI. Assuming a 15\% pre-test probability, these predictors yield a post-test probability of $75.7 \%$ for a positive result with any two or more measures being positive, and a post-test probability of $2.3 \%$

CONCLUSIONS: This study demonstrated the usefulness of a force platform and a self-reported mood scale for predicting

Keywords: Automated neuropsychological assessment metrics, brain acoustic monitor, balance, voice analysis

\section{Introduction}

Traumatic Brain Injury (TBI) is the result of high energy force to the face or skull occurring in a number of ways [1] and categorized according to severity from mild to moderate to severe [2]. Active duty and 
reserve service members are at increased risk for sustaining a mild to moderate TBI compared with their civilian peers [3] in part due to their exposure to blasts and other combat conditions during recent conflicts in Iraq and Afghanistan [4]. Mild traumatic brain injury (mTBI), more commonly known as concussion, can occur from a non-penetrating blast exposure [5]. From the year 2000 to 2006, the U.S. Army experienced a $107 \%$ increase in mTBI incidents, while the civilian sector showed a $46 \%$ decrease for this same time period [6]. TBI is therefore of considerable interest to the military services.

The symptoms of mTBI are far more subtle and difficult to diagnose than moderate to severe TBI. Rapid advances in medical technology are transforming the way military service members are being assessed and treated in our nation's armed forces. Even so, at the time of this study there was no single standard, reliable, objective procedure for evaluating mild traumatic brain injury (mTBI) at Level I trauma centers in the United States [7-8]. Assessment and identification of mTBI is even more difficult in a military theater-of-operation, with limited medical facilities and multiple casualties [9]. However, there is an assemblage of evidence to suggest several emerging technologies may serve as valuable assessment tools to assist in screening individuals who may be experiencing a mTBI.

With blasts contributing to a high number of head injuries in theater, the need for rapid assessment is vital to identify injuries early, so appropriate treatment can commence. Following blast exposure, the initial screening measures in theater for TBI have included assessments using the Military Acute Concussion Evaluation (MACE) [10], the Glasgow Coma Scale (GCS), a neurological assessment (with eye, motor, speech, and balance components), and evaluation of pupillary response, as well as blood pressure, oxygenation, and interview/observation. If no loss of consciousness or alteration of consciousness occurs during administration of the MACE [9], the health care practitioner (typically a medic) may stop and consider other possible causes (other than concussion) for current symptoms. A Concussion Management Algorithm is used to determine the next steps in terms of the level of care needed, such as rest or evacuation to a Combat Support Hospital [9]. These clinical diagnostic tools serve as the 'best we can do under the circumstances', but are open to the interpretation of the individuals administering them. In addition, individuals with a mTBI often do not report their symptoms initially, but may present with symptoms days, weeks, or even months after the incident. Finally, over a third of Iraq veterans thought to have TBI related concussion syndrome had comorbidities of Post-Traumatic Stress Disorder (PTSD) or depression, and the overlapping symptoms of mTBI, PTSD, and depression further confound diagnostics [11]. As a result, a mTBI can exist undiagnosed, putting service members at risk for a second head injury and endangering the military service member and their team.

Often, those who experience a first concussion experience a second concussion. Within the sports medicine realm, this condition is referred to as second impact syndrome and although professionals debate this issue [12], some contend it may produce increased susceptibility for behavioral symptoms, greater diffuse axonal injuries (DAI) [13-18] prolonged neuropsychiatric impairment [19], and later-life cognitive impairment [19-20], including chronic traumatic encephalopathy [21]. In sportsrelated research, sustaining a concussion has been shown to increase the likelihood of sustaining a second concussion [22], possibly due to increased susceptibility.

There are technologies that show potential for identifying mTBI, four of which were targeted for this study. The first two are relatively well known: balance measured with a AMTI OR6-7 force plate and neurocognition and mood using the Automated Neuropsychological Assessment Metric4 (ANAM4) - configured for assessing TBI. The second two selected for study are not as well-known and have less research backing, blood flow comparisons using a Brain Acoustic Monitor (BAM), and voice using Voice Analysis software (VA).

Balance is "the process of maintaining equilibrium and center of gravity within the body's base of support" [23]. Having a problem with balance is the second most frequently self-reported symptom in TBI patients [24]. Multiple investigators have shown postural instability in patients with TBI [25-26]. In fact, Allison has stated that nearly everyone with a TBI has some type of balance problem [27]. While the research clearly indicates balance as an issue among individuals with a TBI, it is not known how long these symptoms persist after the point of injury and which (if any) measures of balance can be used to assist with identifying whether a person has incurred a TBI. That is, most of the research on balance and TBI has focused on rehabilitation techniques to improve balance and recovery [28], rather than using balance to predict who may have a mTBI. 
A constellation of long-lasting symptoms of a TBI is classified as "post-concussion syndrome" (PCS), which includes difficulties with attention and concentration, impaired memory, fatigue, headaches, anxiety, depression, irritability, or sleep disturbances [29]. Hill and colleagues [30] found that over 50\% of the individuals with mTBI included in their study, reported active symptoms of sleep disturbance, irritability, memory problems, and headaches. The Automated Neuropsychological Assessment Metric (ANAM) is a computer-based tool designed "to detect speed and accuracy of attention, memory, and thinking ability" that also includes a mood scale [31]. It includes a number of assessments, which can be activated or deactivated for use with particular populations, illnesses, or injuries. The ANAM4 was specifically configured as a neurocognitive assessment focused on TBI, and it incorporates evaluations of all the symptoms of PCS. The ANAM (including ANAM4) has been administered pre and post soldiers' deployment into harms' way, and to assess patient function among service members with head injuries [32]. The ANAM has also been used, along with other neurocognitive assessments, to set baselines and identify potential head injuries among athletes, using selected tasks to construct the Automated Sports Medicine Battery [33-34]. Its portability and ease-of-use may contribute to its use within war zone theaters of operation [35]. While some studies have found decreased performance on neurocognitive tasks among military with a mild to moderate TBI, others have not [5]. RoebuckSpencer and colleagues [36] showed the highest percentage of post-deployment service members with neurocognitive decline were those who reported active symptoms, as well as having sustained a TBI during deployment.

Correctly identifying a head injury early is important to initiating effective therapy. Active Signal Technologies has developed a sensor system to detect and amplify sound waves within the brain, known as the Brain Acoustic Monitor (BAM) [37-38]. Although not audible by standard measures, the arterial pulse wave produces a predictable deformation of the skull as it enters the brain and can be acoustically measured. The underlying theory for the BAM is that arterial pulse waves produce acoustically detectable, and predictable, changes that can be measured on the forehead. These sounds are altered when there is damage to the cerebrovascular system and differ from those heard at a reference point, such as the radial artery or a finger pulse [37]. In a person with- out a TBI, the cerebrovascular acoustic wave forms are more similar to those at the reference point than in a person experiencing a TBI [38]. Dutton and colleagues examined the effectiveness of using the BAM to identify mTBI in patients with normal and abnormal CT scans, and found the BAM sensitivity to be $100 \%$ with a specificity of $30.14 \%$ [38], while a second study by Dutton and colleagues found a sensitivity of $93 \%$, and a specificity of $14 \%$. Both studies examined individuals early after hospital admission, consequent to their demonstrating anatomic and functional evidence of TBI. From the first study, they concluded the BAM may serve as a useful, additional diagnostic tool, adding important information to selfreports of injury, loss of consciousness, and health care practitioner interviews [38]. From the second study, they concluded early BAM screenings may guide prehospital and emergency room diagnostic and therapeutic decision making, however they suggested further refinements are necessary to improve specificity [39].

Differences in voice and voice analysis results have been demonstrated in severe TBI in terms of irregularity, noise-to-harmonic ratio, and hypernasality [40], sometimes resulting from motor disorders [41]. Speech is currently being explored as a diagnostic tool for mTBI in athletes [42]. Past research investigating voice analysis as a diagnostic tool have included Parkinson's disease [43], Huntington's disease [44], and examination of various health states [45]. While these investigations have resulted in limited success, new technologies and methodologies continue to yield possibilities and the concept of wearable biomarker devices to assist with mTBI identification is appealing. Sound Health's Bioacoustic Biology uses voice, and voice analysis, as representations of an individuals' health and wellbeing, developed after examining the voices of people with similar illnesses or injuries for their similarities in sound frequencies [46]. The theory behind Sound Health's technology is that people with similar health issues produce similar vocal biomarkers. Sound Health's voice analysis (VA) system was used in this study.

As medical evaluation procedures and diagnostic technologies evolve, there is an opportunity to identify injuries expediently and accurately with objective and quantitative measures to avoid susceptibility to further injury and quickly implement appropriate treatment. Moreover, there is a need for rapid, evidence-based, objective diagnostics that can be employed in a combat setting to assist military health 
care providers in screening, identifying, and tracking the symptoms of mTBI in military personnel. At this time, none of the mentioned technologies has a sufficiently strong research backing supporting their use at, or shortly after, the point of injury in a field setting.

The purpose of this research was 1) to examine the usefulness of four technologies in differentiating individuals with and without mild or moderate, service-related TBI, and 2) if found to be useful to then build a clinical prediction rule (CPR) based on the results. The methods and technologies examined included: 1) balance using a force plate and analysis software (AMTI OR6-7-2000) developed by Advanced Mechanical Technology, Inc.), 2) neurocognitive and mood testing using the ANAM4, 3) a comparison of the acoustic wave forms from cerebrovascular blood flow compared with a reference point, using BAM developed by Active Signal Technologies, and 4) voice recordings using voice analysis software developed by the Institute for BioAcoustic Biology and Sound Health.

\section{Methods}

\subsection{Participants}

Prior to participation, all volunteers were briefed on the study, screened by a health care provider to ensure they were medically fit to participate, and signed both informed consent and Health Insurance Portability and Accountability Act (HIPAA) consent documents. Study volunteers were recruited from a population of active duty and retired military service members in the region of a large military medical center. The information presented in this paper is part of a longitudinal study involving three visits spaced several months apart. For the purpose of this paper (examining the diagnostic utility of four technologies) the focus is on the data collected during the first visit. A total of 98 volunteers signed the consent form to participate, with 88 completing the first visit with all four technologies. Those who initially volunteered but did not actually participate in the study did so for a variety of reasons including, ankle injury $(n=1)$, surgery $(n=2)$, and changing their minds about participating $(n=7)$. The two primary groups of volunteers were those with and those without a TBI, following the basic diagnostic criteria presented by Kay and colleagues [47].

\subsection{Procedures}

Military service member volunteers were screened by health care providers (HCPs) in a TBI Clinic to ensure they met the protocol inclusion/exclusion criteria. To be included in the study, volunteers had to be at least 18 years of age; an active duty service member, veteran or retiree; and be able to read and understand English. Volunteers could not have any medical contraindications to study participation (i.e., lower extremity injury or a disorder-other-than-TBI that may affect balance). Potential participants who were diagnosed with TBI, were included if they had a mild to moderate TBI, as the TBI Clinic grouped them together for assessment and rehabilitation purposes (that is, they did not treat mild and moderate cases as separate entities). From this point forward in this paper, mmTBI applies to both those with a minimal or a moderate TBI. Potential participants were excluded if they had a severe TBI. In addition, subjects were excluded if they had ever suffered a previous head injury that resulted in loss of consciousness of more than 20 minutes (other than during military deployment). The TBI Clinic staff used interviews and record reviews, with the following criteria for the diagnosis for TBI: a loss of consciousness $(<30$ minutes), memory loss for events immediately preceding (retrograde amnesia) or following the accident (post traumatic amnesia (PTA) $<24$ hours), changes in mental state at the time of the injury (dazed, disoriented, confused), presence of focal neurological deficits, and a Glasgow Coma Scale score $>13$ [42]. Volunteers completed a demographic questionnaire, including questions about their TBI history. This took approximately 20 minutes. The researchers were blinded to the TBI status of the volunteers to avoid TBI diagnosis from biasing researcher interaction with participants. Therefore, the demographic form was put into a sealed envelope and opened only by two un-blinded researchers who did not interface with the research participants during testing, but later entered their data into the database. Research volunteers were then assessed using the four technologies described below. The testing sequence, if one subject was present was demographics, balance, VA, BAM, and ANAM. If more than one person was present, they were tested simultaneously, each on a different measure, as we had only a single force platform, voice analysis system, and BAM. Multiple subjects could complete the demographic and ANAM4 assessments simultaneously. These arrangements decreased participants' wait time and total time to complete the 
assessments. Participants were encouraged to take breaks and they were permitted to schedule their pre/post assessments over several days, if necessary.

\subsection{Technologies}

\subsubsection{Force platform}

This study examined the predictive ability of various measures of balance in the identification of mTBI. Each volunteer participated in a series of standing balance tests, i.e., measurements of postural sway first with their eyes open and then with vision occluded by wearing a mask over their eyes. The balance tests were performed on an AMTI OR67 force platform using integrated AMTI NetForce software. All stances were performed with the participant wearing socks. We evaluated eight separate positions with eyes open and eyes closed and compared measurements of sway displacement, velocity, and variability between groups across positions to identify a subset of variables that would appropriately discriminate between individuals with and without mTBI. The positions we evaluated can be generally categorized across three descending levels of base of support (BOS): bilateral stance, tandem stance, and single limb stance, and two levels of vision: vision and no vision. The specific balance positions selected were those used frequently in evaluations of balance. They are shown in Table 1 and were performed in the order listed. Use of the force plate yielded 418 pre/post outcome variables. These measures took approximately 45 minutes to complete.

\subsubsection{Automated Neuropsychological Assessment Metric (ANAM)}

Individual cognitive assessments were conducted using the ANAM4 TBI Battery on a laptop computer. This automated battery of tests was developed specifically for use in screening for concussion/TBI [48]. The measures administered as part of the TBI Battery can be seen in Table 2 .

The ANAM was administered twice during the first visit to help account for learning effects and to provide a more reliable set of test scores. The first administration served as a practice session to familiarize the volunteer with the evaluations, whereas data from the second administration (given at the conclusion of the visit) were analyzed.

The ANAM Mood Scale 2 - Revised (AMS-2) is included within the ANAM software. The AMS2 is comprised of 42 mood-related adjectives, rated on a 7-point Likert scale of mood intensity, ranging from 0 to 6 . The lowest verbal anchor is 0 "not at all", midpoint is 3 "somewhat", with a maximum of 6 "very much". The mood adjectives are grouped under seven mood subscales: anxiety (tension/anxiety level), depression (dysphoria), anger (negative disposition), vigor (high energy level), fatigue (low energy level), happiness (positive disposition), and restlessness (motor agitation) [49]. The full ANAM4 battery took approximately 30-45 minutes per administration and yielded 55 pre/post outcome variables.

\subsubsection{Brain Acoustic Monitor (BAM)}

The BAM protocol used for this study closely followed the University of Maryland R. Adams Cowley Shock Trauma Center (STC) protocol using a laptop-based system. The system included a Panasonic Toughbook ${ }^{1}$ with the conditioning electronics located on the base of the laptop, two forehead sensors secured using an elastic headband, and a finger reference sensor (Figs 1-3).

The BAM sensor disks were applied to the front of the participant's forehead close to the volunteer's hairline and centered over the pupils of each eye, under an elastic cloth band. A modified pulse oximeter finger clip was applied to the middle or ring finger, which served as the reference sensor. Acoustic data from the two forehead sensors and from the reference point were then recorded for 10 seconds. This process was repeated a total of five times per visit or until an acceptable reading was obtained, which was indicated on a BAM display. An unacceptable display most often reflects incorrect placement of the sensors. The waveforms are measured in time and frequency domains. In a healthy individual the forehead signals resemble the arterial reference location, while they do not match in someone with a head injury. The BAM system has a signal processing algorithm, with allowable boundaries of divergence. The individual is considered 'healthy' or 'normal' if the readings fall within the boundaries (BAM Negative). They individual is considered suspicious for pathology if the readings fall outside the boundaries (BAM Positive). The system has a red/green display indicating the sensor output results. This assessment produces 7 outcome variables. Testing with the BAM required approximately 10 to 20 minutes [50].

\footnotetext{
${ }^{1}$ Toughbook is registered trademark of Panasonic Corporation
} 
Table 1

Balance Positions

\begin{tabular}{|c|c|c|}
\hline Stance & Description & Image \\
\hline Bilateral Stance & $\begin{array}{l}\text { Feet side by side, apart; arms; eyes } \\
\text { open and closed }\end{array}$ & \\
\hline $\begin{array}{l}\text { Tandem Stance, right foot } \\
\text { in front of left }\end{array}$ & $\begin{array}{l}\text { Right foot in front of left; arms at } \\
\text { sides; eyes open and closed }\end{array}$ & \\
\hline $\begin{array}{l}\text { Tandem Stance, left foot } \\
\text { in front of right }\end{array}$ & $\begin{array}{l}\text { Left foot in front of right; arms at } \\
\text { sides; eyes open and closed }\end{array}$ & \\
\hline $\begin{array}{l}\text { Tandem Stance, dominant } \\
\text { foot forward }\end{array}$ & $\begin{array}{l}\text { Dominant foot in front on } \\
\text { non-dominant foot, arms at sides; } \\
\text { eyes open }\end{array}$ & \\
\hline Romberg Test & $\begin{array}{l}\text { Bilateral stance with ankles together, } \\
\text { arms crossed, fingers touching } \\
\text { shoulder; eyes open and closed }\end{array}$ & \\
\hline $\begin{array}{l}\text { Military Academy Stance } \\
\text { Test, (MAST) right leg }\end{array}$ & $\begin{array}{l}\text { One-legged stance, balancing on } \\
\text { right leg, left leg lifted and bent in } \\
\text { a comfortable position, arms at } \\
\text { sides; eyes open and closed }\end{array}$ & \\
\hline $\begin{array}{l}\text { Military Academy Stance } \\
\text { Test, (MAST) left leg }\end{array}$ & $\begin{array}{l}\text { One-legged stance, balancing on left } \\
\text { leg, right leg lifted and bent in a } \\
\text { comfortable position, arms at } \\
\text { sides; eyes open and closed }\end{array}$ & \\
\hline
\end{tabular}

\subsubsection{Voice Analysis (VA)}

Voice data were gathered using a Sound Recorder [51], and analyzed using proprietary software developed by the Institute for BioAcoustic Biology and Sound Health [46]. A uni-directional condenser microphone (SAMSON C01U USB) was connected to a laptop computer. The laptop computer was configured with BioAcoustic Biology and Sound Health vocal analysis software and a calibrated sound card [46]. The sound recorder settings included the sound playback default device set at Sigma Tel Audio, the sound recording default device set at SAMSON C01U, the volume set at maximum, and the MIDI Playback set at Microsoft GS Wavetable SW Synth.
Three recordings were made, with each recording being 30 seconds long. The three recordings included one recording of the volunteer reading a pre-selected paragraph, one talking about personal physical health, and one talking about a mundane subject, such as what they had for breakfast or lunch.

The recorded vocal sounds were converted to numeric data using properties of frequency (hertz/cycles per second) and amplitude (decibel) by means of a Fast Fourier Transform. This translates the vocal data to numeric data creating a digital graph that represents the vocal frequencies using a range of 0-1000 cycles per second, depicting a grid of 0 to 60 on the $Y$ axis and 0 to 1000 along the $\mathrm{X}$ axis. Each volunteer's voice analysis included 24 
Table 2

Automated Neuropsychological Assessment Metric components administered

\begin{tabular}{|c|c|}
\hline Assessment & Description \\
\hline Demographics & $\begin{array}{l}\text { Demographic information such as } \\
\text { age and gender }\end{array}$ \\
\hline Sleepiness Scale & $\begin{array}{l}\text { Measure of user's current state of } \\
\text { energy-fatigue level }\end{array}$ \\
\hline Mood Scale & $\begin{array}{l}\text { Anxiety (anxiety level), and } \\
\text { restlessness (motor agitation) }\end{array}$ \\
\hline Simple Reaction Time & Index of visuo-motor response timing \\
\hline $\begin{array}{l}\text { Code Substitution- } \\
\text { Learning }\end{array}$ & $\begin{array}{l}\text { Index of visual search, sustained } \\
\text { attention, and encoding }\end{array}$ \\
\hline $\begin{array}{l}\text { Procedural Reaction } \\
\text { Time }\end{array}$ & $\begin{array}{l}\text { Reaction time and processing } \\
\text { efficiency associated with simple } \\
\text { mapping rules }\end{array}$ \\
\hline $\begin{array}{l}\text { Mathematical } \\
\text { Processing }\end{array}$ & $\begin{array}{l}\text { Basic computational skills, } \\
\text { concentration, and working } \\
\text { memory }\end{array}$ \\
\hline Matching to Sample & $\begin{array}{l}\text { Spatial processing and visuo-spatial } \\
\text { working memory }\end{array}$ \\
\hline $\begin{array}{l}\text { Code substitution - } \\
\text { delayed }\end{array}$ & Memory (delayed) \\
\hline Simple Reaction time & Index of visuo-motor response timing \\
\hline
\end{tabular}

base frequencies identified by the software as being "in stress" [52]. These 24 frequencies, along with their 24 'reciprocal' values were then compared with a series of frequencies identified by the Institute for BioAcoustic Biology and Sound Health using their proprietary Army Compare database, as being associated with TBI injuries. While not true mathematical reciprocals, the 'reciprocal' values are mathematically related to the base frequencies via proprietary software calculations [52]. For the purpose of this initial examination, the number of each individual's 48 values (frequencies + reciprocals) that also appeared in the Army Compare database (i.e. were thought to be associated with TBI) were used for the data analysis. Twelve outcome measures were produced. Data collection for voice analysis took approximately five to ten minutes.

\subsection{Data analysis}

Univariate analyses of between-group mean differences served as initial tests to determine whether measurements from the clinical instruments discriminated between volunteers with vs. without mmTBI. These analyses were performed with analysis of covariance (ANCOVA) using age, gender, and length of military service to adjust for between-group differences on these variables. The alpha level for the preliminary for potential predictors was relaxed to .10 to reduce Type II error probability. For force plate variables of center of pressure, the magnitudes of movement (regardless of direction) were used.

Multivariate binary logistic regression analysis was used to further reduce the set of predictor variables and to derive a multivariate model that eliminated redundant or substantially correlated predictors, or any predictors that did not contribute meaningfully to the multivariate prediction. Potential predictors that yielded $p$-values $<.10$ from the ANCOVAs were entered into the logistic regression analyses using a forward stepwise procedure. Probability levels were set to .05 for entry and .10 for removal for each step of model development. Original (non-dichotomized) values for each potential predictor were entered into the logistic regression analysis. In order to avoid over-fitting the model, an a priori decision was made to limit the number of retained predictors such that there were at least 10 subjects with mmTBI per each variable in the final model [53].

Predictors in the final model were individually characterized for diagnostic accuracy by first dichotomizing the scales using cut-scores determined from the Youden index applied to tables of coordinate points from receiver operating characteristic (ROC) curve analyses. Dichotomized predictors were then combined into a multivariate CPR with levels defined by the number of predictors considered positive
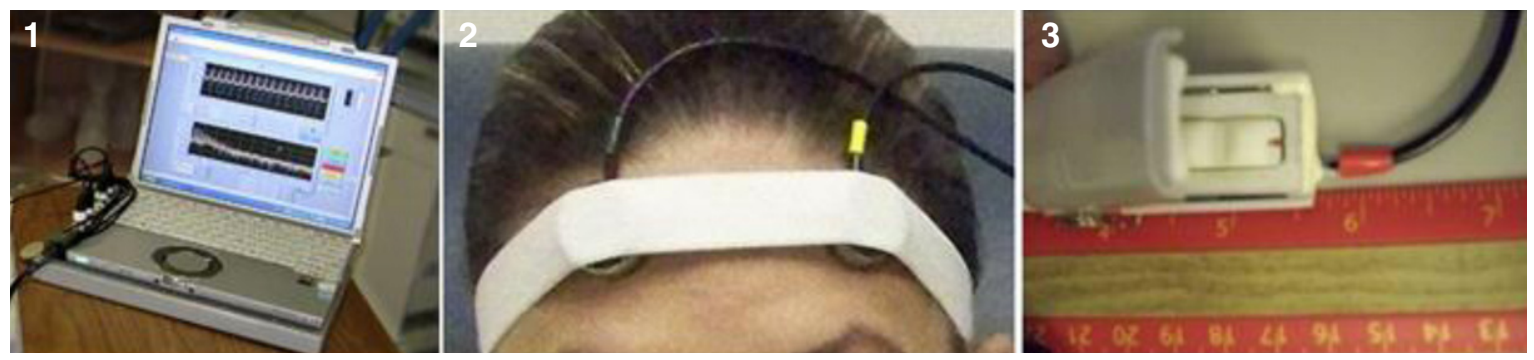

Fig. 1-3. BAM equipment (Left to Right): Panasonic Toughbook, forehead sensors with headband, and finger reference sensor. 
(above or below the cut score). Each level of the CPR (at least 1 test positive; at least 2 tests positive, etc.) was characterized with calculation of sensitivity, specificity, Positive Likelihood Ratio (PLR), and Negative Likelihood Ratio (NLR). The prevalence of individuals with mmTBI returning from the conflicts in Iraq and Afghanistan has been estimated to be 15\% [54-55], therefore, we used this estimate for our pretest probability. Specifically, for our purposes, this was assumed to be the probability that a Soldier who had sustained an injury in theater and was transferred to a tertiary care facility would have a mmTBI prior to any testing occurring. For each level of positive predictors in the CPR, post-test probabilities for the diagnosis of mmTBI for positive and negative CPR results were computed based on a generalized pretest probability (using a prevalence of 15\%) together with the PLRs and NLRs.

\section{Results}

Among the 98 volunteers enrolled in the study, 88 provided reasonably complete data for variables derived from force plate, ANAM, BAM, and VA data collection instruments. Of those completing the study, 35 (39.8\%) had a diagnosis of mmTBI. Participants with mmTBI $(30.7 \pm 7.5$ years $)$ were significantly younger $(p<.001)$ than those with no TBI (39.1 \pm 10.2 years). Likewise, participants with mmTBI had significantly fewer $(p<.001)$ years of military service $(9.1 \pm 7.1$ years) than those with no TBI (16.1 \pm 8.4 years). Gender proportions were also significantly different between groups $(p=.002)$ : in the mmTBI group only 1 (2.9\%) of 35 subjects was female; in the no TBI group 15 (28.3\%) of 53 participants were female. Demographic variables are summarized for each group in Table 3. Among the
mmTBI subjects in our study $(n=26)$, the mean time since injury at time of data was $207 \pm 248$ days (range 15 to 1088 days).

From a total of 492 available variables (418 force plate variables, 55 ANAM variables, 7 BAM variables, and 12 voice variables), 50 potential predictors had significantly different mean values at a relaxed alpha level (.10) comparing the mmTBI group to the no TBI group, and controlling for age, gender, and years of military service. Among those variables showing univariate groupwise discrimination were 40 force plate variables (Table 4), 8 ANAM variables, 1 BAM variable, and 1 voice variable (Table 5). Of these 50 potential predictor variables, four variables were eliminated from further consideration because of missing data due to human and/or recording failures. Another seven variables were eliminated because of between-group mean differences showing paradoxically better balance in the mmTBI group.

This left 39 variables which were entered into the multivariate stepwise logistic regression analysis based on 83 subjects ( 32 with mmTBI) with complete data for all 39 entered predictors. The stepwise procedure retained 3 predictors: 1) ANAM4 mean of ratings for the Vigor adjectives from the Mood Scale; 2) absolute value of average center of pressure $x$-axis $(\mathrm{cm})$ with left foot in front of right, eyes open; 3 ) average displacement along y-axis $(\mathrm{cm})$ on right leg, eyes open. This model correctly classified $64(77.1 \%)$ of 83 subjects (Table 6), and was statistically significant $(p<.001)$, with the 3 predictors explaining $37.5 \%$ of the variance in TBI status. Sensitivity (precision or True Positive Rate) is the ability to correctly identify an individual with mmTBI. Specificity (True Negative Rate) is the ability to correctly identify those who do not have a mmTBI, based on their negative scores.

The Hosmer-Lemeshow statistic established reasonable goodness of fit for the model $(p=.684)$.

Table 3

Characteristics of Subjects Completing the Study

\begin{tabular}{lccc}
\hline Subject characteristic & $\begin{array}{c}\text { mmTBI Group } \\
(n=35)\end{array}$ & $\begin{array}{c}\text { No TBI Group } \\
(n=53)\end{array}$ & P \\
\hline Age, years & $30.7(7.5 \%)$ & $39.1(10.2 \%)$ & $<.001$ \\
Women, no. (\%) & $1(2.9 \%)$ & $15(28.3 \%)$ & 0.002 \\
Caucasian Ethnicity, no. $(\%)$ & $21(60.0 \%)$ & $38(71.7 \%)$ & 0.382 \\
Active duty Army, no. $(\%)$ & $28(80.0 \%)$ & $43(81.1 \%)$ & 0.886 \\
Years current military service & $9.1(7.1)$ & $16.1(8.4)$ & $<.001$ \\
How many times deployed & $2.1(1.4)$ & $1.6(1.4)$ & 0.106 \\
Years since return from last deployment & $1.2(1.7)$ & $3.3(4.6)$ & 0.004 \\
\hline
\end{tabular}

Values presented in the two group columns are mean (SD) unless otherwise specified. The $p$ values are from unpaired $t$-tests for continuous-scale variables and from Chi-square tests or Fisher's Exact test (where needed) for categorical variables. 
Table 4

Force plate variables with significantly different means (adjusted for age, gender, and years of military service) for mmTBI group vs. no TBI group

\begin{tabular}{|c|c|c|c|c|c|c|c|}
\hline & & Romberg & Sharpened & Tandem & & MA & \\
\hline & Eyes & & KVח & $\frac{\mathrm{L}}{\mathrm{L}}$ & $\overline{\mathrm{R}}$ & $\mathrm{L}$ & $\mathrm{R}$ \\
\hline Average CPOX & $\begin{array}{l}\text { Open } \\
\text { Closed }\end{array}$ & & $\mathrm{X}^{\mathrm{b}}$ & $\mathrm{X}^{* *}$ & & & \\
\hline Average CPOY & Open & & $\mathrm{X}^{\mathrm{b}}$ & $\mathrm{X}$ & $\mathrm{X}$ & $X^{a}$ & \\
\hline & Closed & & $\mathrm{X}^{\mathrm{b}}$ & $\mathrm{X}$ & $\mathrm{X}$ & & \\
\hline Maximum COPX & $\begin{array}{l}\text { Open } \\
\text { Closed }\end{array}$ & & & & & & \\
\hline Maximum COPY & $\begin{array}{l}\text { Open } \\
\text { Closed }\end{array}$ & $\mathrm{x}$ & & $\mathrm{x}$ & & $\mathrm{X}^{\mathrm{a}}$ & $\mathrm{X}$ \\
\hline Minimum COPX & $\begin{array}{l}\text { Open } \\
\text { Closed }\end{array}$ & & & & & & \\
\hline Minimum COPY & $\begin{array}{l}\text { Open } \\
\text { Closed }\end{array}$ & $\begin{array}{l}\mathrm{X} \\
\mathrm{X}\end{array}$ & & & & & \\
\hline SD COPX & $\begin{array}{l}\text { Open } \\
\text { Closed }\end{array}$ & $\mathrm{x}$ & & & & & \\
\hline SD COPY & $\begin{array}{l}\text { Open } \\
\text { Closed }\end{array}$ & $\begin{array}{l}\mathrm{X} \\
\mathrm{X}\end{array}$ & & & & $\mathrm{x}^{\mathrm{a}}$ & $\mathrm{X}$ \\
\hline Displacement COPX & $\begin{array}{l}\text { Open } \\
\text { Closed }\end{array}$ & $\mathrm{X}$ & & & & & \\
\hline Displacement COPY & $\begin{array}{l}\text { Open } \\
\text { Closed }\end{array}$ & $x$ & & & & $\mathrm{X}^{\mathrm{a}}$ & $\mathrm{X}^{* *}$ \\
\hline Radial displacement COP & Open & $1 \times$ & & & & & $\mathrm{x}$ \\
\hline SD Radial displacement COP & $\begin{array}{l}\text { Open } \\
\text { Closed }\end{array}$ & $\begin{array}{l}A \\
X \\
X\end{array}$ & & & & & \\
\hline 95\% Ellipse Area & $\begin{array}{l}\text { Open } \\
\text { Closed }\end{array}$ & $\begin{array}{l}X \\
X\end{array}$ & & & & & $\begin{array}{l}\mathrm{X} \\
\mathrm{X}\end{array}$ \\
\hline 95\% SD Major & $\begin{array}{l}\text { Open } \\
\text { Closed }\end{array}$ & $\mathrm{X}$ & & & & $\mathrm{x}$ & $\mathrm{X}^{\mathrm{b}}$ \\
\hline 95\% SD Minor & $\begin{array}{l}\text { Open } \\
\text { Closed }\end{array}$ & $\mathrm{X}$ & & & & & $\mathrm{X}$ \\
\hline Velocity & $\begin{array}{l}\text { Open } \\
\text { Closed }\end{array}$ & & & & & $\begin{array}{l}X^{a} \\
X^{a}\end{array}$ & \\
\hline
\end{tabular}

$\mathrm{L} / \mathrm{R}$ refers to the foot placed in front during tandem stance or the foot the subject stood on.

Eyes Open/Closed refers to visual condition for each test.

$\mathrm{COP}=$ Center of Pressure

$\mathrm{X}, \mathrm{Y}$ refer to $\mathrm{X}$ - and Y-axes (side-to-side and front-to-back, respectively).

** Retained as a predictor in the logistic regression model.

${ }^{a}$ Not entered into logistic regression because of a counterintuitive direction of difference: mmTBI subjects showing less excursion or less variability or lower velocities than those without TBI.

${ }^{\mathrm{b}}$ Not entered into logistic regression because of too many missing data points.

Adjusted odds ratios were .44 (95\% CI.28 to.67) for the mean of ratings for the Vigor adjectives in the ANAM4 Mood Scale, 2.38 (95\% CI 1.16 to 4.91) for absolute value of average center of pressure $\mathrm{x}$-axis $(\mathrm{cm})$ with left foot in front of right eyes open, and 10.10 (95\% CI 1.62 to 62.95) for average displacement along y-axis $(\mathrm{cm})$ with right leg, eyes open. Areas under the ROC curves for the three retained predictors were as follows: 0.74 (95\% CI 0.64 to 0.84 ) for the ANAM4 mean of ratings for the Vigor adjectives from the Mood Scale, .63 (95\% CI 0.51 to 0.75 ) for absolute value of average center of pressure $\mathrm{x}$-axis $(\mathrm{cm})$ with left foot in front of right eyes open, and .57 (95\% CI 0.44 to 0.70 ) for average displacement along y-axis $(\mathrm{cm})$ with right leg, eyes open. Cut scores determined with the Youden Index method, defining positive test results for each of the retained predictors, were $<2.42$ points, $>1.44 \mathrm{~cm}$, and $>1.00 \mathrm{~cm}$, respectively.

Diagnostic accuracy profiles for each level of the 3item CPR, using all predictors in combination, based on $n=86$ with complete data for all 3 predictors, are presented in Table 6 . Table 7 shows the three tests for mmTBI, including the score on each measure which denotes when it is positive for predicting a mmTBI. 
Table 5

ANAM, BAM, and VA variables with significantly different means (adjusted for age, gender, and years of military service) for mmTBI group vs. no TBI group

Potential predictor

ANAM matching to sample mean response time (for all correct $\&$ incorrect responses)

ANAM matching to sample throughput (number of correct responses per unit of available response time [=NumCorr/((NumCorr +

NumInc)* MeanRT + NumLapse*Timeout)])

ANAM mean of ratings for the VIGOR adjectives from the Mood Scale **

ANAM mean of ratings for the HAPPINESS adjectives from the Mood Scale

ANAM simple reaction time mean response time (for all correct $\&$ incorrect responses)

ANAM simple reaction time throughput (number of correct responses per unit of available response time

ANAM simple reaction time (repeated) mean response time (for all correct \& incorrect responses)

ANAM simple reaction time (repeated) throughput (number of correct responses per unit of available response time

BAM: Right Side amplitude from brain acoustic monitoring

VA: Sum of frequencies within a subjects 24 identified frequencies that fall out of normal range AND were previously identified in the 'Army Compare' program as being related to TBI

** Retained as a predictor in the logistic regression model.

Table 6

Multivariate diagnostic accuracy for levels of 3 -item CPR, based on $n=86$ with complete data

\begin{tabular}{|c|c|c|c|c|c|c|}
\hline $\begin{array}{l}\text { Level of } \\
\text { 3-Item CPR }\end{array}$ & Sensitivity & Specificity & PLR & NLR & $\begin{array}{c}\text { Post-test } \\
\text { Probability if } \\
\text { Criterion Met } \\
\text { for Respective } \\
\text { Level* }\end{array}$ & $\begin{array}{c}\text { Post-test } \\
\text { Probability if } \\
\text { Criterion Not } \\
\text { Met for Respective } \\
\text { Level }{ }^{*}\end{array}$ \\
\hline All 3 tests positive** & - & - & - & - & - & - \\
\hline At least 2 tests positive & 0.33 & 0.98 & 17.67 & 0.68 & $75.7 \%$ & $10.7 \%$ \\
\hline At least 1 test positive & 0.91 & 0.68 & 2.83 & 0.13 & $33.3 \%$ & $2.3 \%$ \\
\hline
\end{tabular}

PLR = Positive Likelihood Ratio; NLR = Negative Likelihood Ratio * Post-test probabilities are computed from a pre-test probability of $15 \%$ and the respective likelihood ratios ${ }^{* *}$ No subjects in the study had all 3 tests positive.

Table 7

Three variable scores that positively predict mmTBI

\begin{tabular}{lc}
\hline Test & $\begin{array}{c}\text { Positive if } \\
\text { score is: }\end{array}$ \\
\hline $\begin{array}{l}\text { ANAM mean of ratings for the Vigor adjectives } \\
\text { from the Mood Scale }\end{array}$ & $<2.42$ \\
$\begin{array}{l}\text { Absolute value of average center of pressure x-axis } \\
(\mathrm{cm}) \text { with left foot in front of right, eyes open }\end{array}$ & $>1.44$ \\
$\begin{array}{l}\text { Average displacement along y-axis }(\mathrm{cm}) \text { with right } \\
\text { leg, eyes open }\end{array}$ & $>1.00$ \\
\hline
\end{tabular}

\section{Discussion}

The purpose of this research was to examine the usefulness of four technologies in differentiating military service members with and without mild or moderate, service-connected TBI, and to build a clinical prediction model based on the results. Our statistical analysis of four technologies, the AMTI force plate, ANAM4, the BAM, and VA resulted in two of the four devices being helpful in predicting TBI: the AMTI Force plate for two measures and the ANAM4 mood subscale for vigor. A clinical prediction model was developed.

\subsection{Balance}

Postural stability is defined as maintaining one's center of mass within the BOS [56], and it has been established that postural sway increases in conditions when the BOS is reduced [57-58] and in conditions without vision [59]. Our balance conditions ranged across a continuum from simple (bilateral stance, feet apart, eyes open) to difficult (single limb stance eyes closed) [60]. We observed that differences in sway velocity and variability inconsistently discriminated between subjects with mmTBI and controls across all balance conditions and thus, were not included in our final model. Sway velocity and variability have previously been reported to demonstrate poor test-retest reliability [61], and may provide inadequate discrimination of population differences. Frontal and sagittal plane sway displacement were found to be significantly predictive of a diagnosis of mmTBI) with left foot in front of right eyes open, and with right leg, eyes open respectively. In previous investigations comparing mmTBI patients and controls, postural stability has not consistently been shown to be diminished among mmTBI patients during quiet stance 
or under conditions of diminished BOS and vision [25, 62-63]. Kaufman and colleagues [25] found that mmTBI patients demonstrated diminished postural stability only during a single sway referenced condition on the sensory organization test, but no deficits were seen during quiet stance with the eyes open or closed. Slobounov et al. [63] reported that athletes who sustained a mmTBI demonstrated no deficits in measures of postural sway while performing bilateral stance with eyes open and closed 30 days after injury. In contrast, Findling and colleagues [62] found that mmTBI patients with whiplash demonstrated greater postural sway during bilateral stance conditions on a firm surface with eyes open and closed compared to controls and patients with whiplash alone, as well as increased sway during unilateral stance on a firm surface with eyes closed compared to controls. Our results partially agree with these studies, showing postural stability was diminished among mmTBI participants [25-63], and differed from studies that either showed postural sway was not diminished [62-63] or other measures of postural sway were diminished [25-63]. Differences in postural deficits observed in this study compared to those reported elsewhere, may have occurred for several reasons. First, these differences may be due to the duration of injury in our mmTBI participants. Participants in the current study had sustained a mmTBI an average of 6.9 months prior to data collection, with a range between 15 days and 3 years. Kaufman and colleagues [25] reported on patients with an average injury duration of 2.8 years, while those described by Slobounov and colleagues [56] had sustained an injury within 30 days of testing. Findling et al. [62] described deficits in patients with symptoms of at least 6 months duration. Another potential reason for the difference in our findings compared to those reported elsewhere may be due to methodological differences in the studies. Kaufman et al. [25] reported body sway measured during the sensory organization test conducted on the Equitest device, which provides summarized output of postural sway. Additionally, while other studies have used force plate measurements, their methodologies differ from those of the current study in the number of foot positions and visual conditions evaluated [25-62-64]. Finally, the mechanism of injury may impact balance, such as blast injuries vs. physical impact injuries.

Should future research uphold these findings, ruggedizing a force platform for military field use will still be a challenge. Force platforms need to be stable and require a consistent, flat surface perhaps more easily implemented in a garage or motor pool than a field hospital. In addition, they (and the computer technology they use) are sensitive to dirt, dust, and sand, often found in places of military deployment, such as recent altercations in Iraq and Afghanistan.

\subsection{ANAM}

Participants' rankings of vigor adjectives on the Profile of Mood States remained in the model. The vigor subscale addresses feelings related to a persons' energy level and includes adjectives such as energetic, lively, alert, spirited, active, and vigorous. Those with mean scores equal to or below 2.42 were more likely to have a mmTBI diagnosis. While this would appear to be consistent with findings of fatigue among those who have incurred a traumatic brain injury, as found by others [65-69], in our study the subscale for fatigue was not predictive of mmTBI. Post TBI fatigue has been found to be related depression, pain, and sleep problems [68]. Identifying vigor as a predictor in our model might also appear to be related to research demonstrating that individuals with a TBI are more likely to have depressive symptoms, than individuals without a TBI [69-73]. However, in our study the subscale for depression was not predictive of mmTBI.

Individuals who have experienced a TBI often have difficulty sleeping [74-75], which may be related to vigor. However, our study did not demonstrate differences in sleepiness between those with and without a mmTBI. Castriotta and colleagues found increased sleep difficulties among those with a TBI, and did not find differences in self-ratings using the Profile of Mood States (POMS), which does have a vigor subscale [76]. Also, although Johnson (et al.) found a correlation between an automated mood scale vigor subscale and the POMS vigor subscale of .64, and a correlation of .76 with the subscale of "energetic arousal" for the Dundee Stress State Questionnaire, the differences between these assessments may be sufficient to yield contradictory research results [49]. While our measure of fatigue used Likert Scale ratings for six adjectives (lazy, inactive, tired, weary, sluggish, and drowsy), others used descriptions that were paired with activities. For example, Ziino and Ponsford used a visual analogue scale for fatigue, a nine-point fatigue severity rating that linked fatigue with behavioral indicators (ex. fatigue interferes with my physical functioning), and a causes of fatigue scale that linked activities with fatigue (concentrating, exercising, etc.) [67]. Johansson and colleagues 
[65] used a 15 item questionnaire that also associated common activities to symptoms associated with mental fatigue, such as sensitivity to stress, irritability, and sensitivity to light. Thus, the differences between the scales themselves may contribute to the differences in our findings, from those of other studies.

Since only the vigor subscale of adjectives was identified in the clinical prediction model, this does not necessarily support the use of the entire ANAM4 or the whole ANAM4 mood scale for screening for mmTBI. Should future research uphold the vigor subscale as being predictive of mmTBI, then that single scale could easily be used.

\section{3. $B A M$}

While prior research is promising for use of the BAM in identifying concussion, positive findings occurred among individuals whose head injury was very recent, for example after hospital admission from an Emergency Room visit [38]. Participants in this study had much longer time passage since their original injury (mean of 6.9 months), with a large standard deviation (over 8 months) and range. This may explain the lack of positive findings with the BAM in this study, as the cerebrovascular damage may have already been arrested in our population. Revisions to the technology now allow use of the BAM in under ten minutes [77], thus it is quick and could provide additional information immediately, or closely following injury. Further research is suggested to determine usability in a military field situation, and how long post injury the BAM remains sensitive to cerebrovascular injury.

\subsection{Voice analysis}

Few research studies have been published on voice analysis and TBI and while results are conflicting, it appears that laryngeal dysfunction coincides with severe TBI. Theodoros and Murdoch found laryngeal hyperfunction [78], while McHenry found laryngeal hypofunction and breathiness among individuals with severe TBI [79]. A second study by McHenry found the most frequently occurring abnormality among those with severe TBI to be amplitude perturbation, followed by voice turbulence index, with nearly a quarter of the research volunteers showing abnormalities in all of the five voice measurements [80]. While interest remains in identifying and using speech acoustic features as biomarkers for portable diagnostic tools for TBI [81], the field remains in its' infancy. Research has focused on severe TBI. The lack of positive findings in this study are likely due to our population, who had mild to moderate TBI rather than severe TBI. Additional studies are needed to identify acoustic biomarkers that are consistent for each level of TBI.

Overall, the observed differences in this study compared to other studies may involve the mechanism of injury. Multiple authors have suggested that mTBI associated with blast injury may produce symptoms that differ from those of blunt force trauma, such as a motor vehicle accident, fall, or sports-related impact [16, 82-84]. Specifically, these injuries may produce a more diffuse axonal injury, rather than the focal injuries associated with coup-countercoup mechanisms seen in other injury mechanisms [82].

\section{Conclusions and recommendations}

This study identified a regression equation predicting those who did, and did not, have a mmTBI, consisting of three measures one from a mood scale and two from use of a force platform. From this

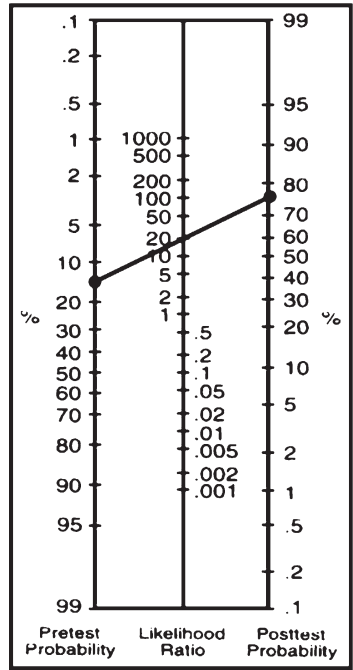

A

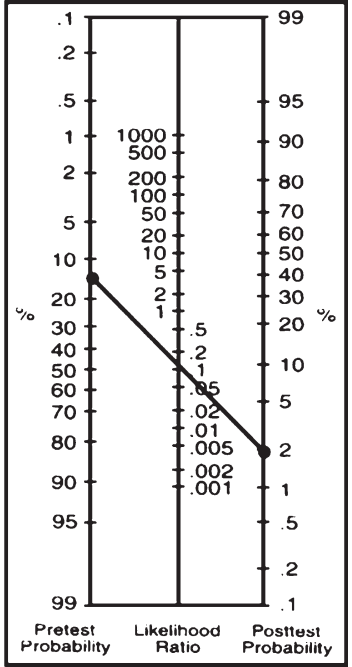

B
Fig. 4. Likelihood ratio nomograms* showing post-test probabilitiesłfor: (A) a positive result with any $2+$ tests positive on the 3 -item CPR, and for (B) a negative result with 0 tests positive.* [55, 84]. Copyright (C1975, Massachusetts Medical Society. All rights reserved. $\ddagger$ Assumes $15 \%$ pre-test probability for mild to moderate TBI. 
equation, a clinical prediction rule was developed to identify patients with a mild to moderate traumatic brain injury. The results indicate that the presence of at least two of the three clinical measures: the ANAM4 vigor subscale score $\leq 2.42$, frontal plane sway $\geq 1.44 \mathrm{~cm}$ during tandem stance with eyes open and the left foot forward, and sagittal plane sway $\geq 1 \mathrm{~cm}$ while standing on the right foot with eyes open increases the likelihood of the patient having a mmTBI from $15 \%$ to $76 \%$. The absence of all three of these predictors decreases the patient's likelihood of having a mmTBI to approximately $2 \%$. That is, a subject with positive results on any two or on all 3 tests would shift them from a pre-test probability of $15 \%$ to a post-test probability of $75.7 \%$ based on a PLR of 17.67. A subject with negative results on all 3 tests in the CPR would shift them from a pre-test probability of having mmTBI from $15 \%$ to a posttest probability of $2.3 \%$ based on a NLR of 0.13 . These results demonstrate the increase in the ability to correctly identify those with a mmTBI and rule out those who do not have a mmTBI. Likelihood ratios of these magnitudes are characterized as sufficient to "... generate large and often conclusive changes in pretest to posttest probability" for a positive test result, and "... moderate shifts in pretest to posttest probability" for a negative test result [85]. That is, the model performs better at ruling in mild to moderate TBI than ruling out the condition. Figure 4 provides a quick visual reference showing the changes in pretest probability to post-test probability, summarizing our findings.

Recommendations include future studies of military service members exposed to blast, much closer to the time of initial injury. Such studies may include balance, mood scales, and the BAM, as well as other, perhaps newer technologies and tools, as they become available. The balance platform is an example an assessment that is very sensitive to balance, perhaps indicating the need for development of more sensitive measures of underlying sensorimotor and executive functioning deficits associated with TBI, such as oculomotor control [87], working memory [88], or other deficits. In addition, research suggestions include examining balance and vigor measures for their prediction consistency with a larger population, both closer to the time of injury and over the course of recovery. While voice analysis shows promise in other studies, it appears more research is required prior to examining its' use with mild to moderate TBI.

\section{Limitations}

This study was limited by the small number of subjects who completed the study. It is possible that a greater number of variables would have been included in the model, given a higher number of participants. Additionally, the study was limited by the inclusion of only subjects with mmTBI being seen in a tertiary care facility, and who were enrolled an average of 207 days (6.8 months) following injury. Therefore, the accuracy of this prediction rule to identify mmTBI in acutely injured individuals remains untested at this time. However, persistent deficits in balance following mTBI have been previously reported [26-8], and the results of our study, if subsequently validated, should help to inform decisions about prognosis and treatment considerations in individuals exposed to blast injury where diagnosis may have been delayed or not established due to comorbid conditions. McGinn and colleagues have proposed methodological guidelines for clinical prediction rules, suggesting that prospective validation and impact analyses should follow initial derivation studies before a clinical prediction rule is considered ready for widespread clinical use [89]. Therefore, future research with an independent sample of subjects should be conducted to ascertain the prediction rule's validity.

\section{Conflict of inetrest}

None to report.

\section{References}

[1] Halliday A. Pathophysiology. In D Marrion (Ed), Traumatic Brain Injury. 1999; 29-38.

[2] Cooper D, Chau P, Armistead-Jehle P, Vanderploeg R, Bowles A. Relationship between mechanism of injury and neurocognitive functioning in OEF/OIF service members with mild traumatic brain injuries. Military Medicine. 2012; 177(10):1157-60.

[3] Cook G, Hawley J. A review of mild traumatic brain injury diagnostics: Current perspectives, limitations, and emerging technology. Military Medicine. 2014;179(10):1083-9.

[4] Elder, G.A. Update on TBI and cognitive impairment in military veterans. Current Neurology and Neuroscience Reports, 15:68.

[5] Gu M, Kawoos U, McCarron R, Chavko M. Protection against blast-Induced traumatic brain injury by increase in brain volume. BioMed Research International. 2017; 2017:1-10. in B, Kane R, Schwab K. Performance on the 
Automated Neuropsychological Assessment Metrics in a Nonclinical Sample of Soldiers Screened for Mild TBI After Returning From Iraq and Afghanistan. Journal of Head Trauma Rehabilitation. 2009;24(1):24-31.

[6] Ivins B, Kane R, Schwab K. Performance on the automated neuropsychological assessment metrics in a nonclinical sample of soldiers screened for mild TBI after returning from Iraq and Afghanistan. Journal of Head Trauma Rehabilitation. 2009;24(1):24-31.

[7] Williams V, Pichelmann M, Atkinson J. Diagnoses of traumatic brain injury not clearly associated with deployment, active component, U.S. Armed Forces, 2001-2016. Msmr. 2017;24(3):32-35

[8] Blostein P. Jones, S. Identification and evaluation of patients with mild traumatic brain injury: Results of a national survey of level 1 trauma centers. Journal of Trauma. 2000;55(3):450-3.

[9] Marshall K, Holland S, Meyers K, Martin E, Wilmore M, Grimes C. Mild traumatic brain injury screening, diagnosis, and treatment. Military Medicine. 2012;177(8 Suppl):6775.

[10] DVBIC. Military Acute Concussion Evaluation (MACE) pocket cards. Available at http://wwwjsomonline.org/TBI/ MACE_revised_2012.pdf; accessed November 21, 2014; 2012

[11] Vasterling JJ, Verfaellie M, Sullivan KD. Mild traumatic brain injury and posttraumatic stress disorder in returning veterans: Perspectives from cognitive neuroscience. Clin Psychol Rev. 2009; 29(8):674-84.

[12] McCrory P. Does second impact syndrome exist? Clinical Journal of Sport Medicine. 2001;11(3):144-9.

[13] Cantu R. Athletic concussion. Neurosurgery. 2007;60(6): 963-4.

[14] Cantu R. Second impact syndrome: Concussion and second injury brain complications. Yearbook of Sports Medicine. 2011;2011:26-8.

[15] Collins M, Lovell M, Iverson G, Cantu R, Maroon J, Field M. Cumulative effects of concussion in high school athletes. Neurosurgery. 2002;51(5):1175-81.

[16] Durand P, Adamson G. On-the-Field Management of Athletic Head Injuries. Journal of the American Academy of Orthopaedic Surgeons. 2004;12(3):191-5.

[17] Warren, W. L., \& Bailes, J. E. (2000). On the field management of athletic head and neck injuries. In R. Cantu (Ed.), Neurologic athletic head and spine injuries (pp. 52-65). Philadelphia, PA: W.B. Saunders.

[18] Wetjen, N. M., Pichelmann, M., \& Atkinson, J. L. (2010). Second impact syndrome: Concussions and second injury brain complications. Journal of the American College of Surgeons, 211(4), 553-557. Winter, D. A. (1990). Biomechanics and motor control of human movement. (2nd ed.). New York: Wiley.

[19] Moser R, Schatz P, Jordan B. Prolonged Effects of Concussion in High School Athletes. Neurosurgery. 2005;57(2):300-6.

[20] Guskiewicz K, Marshall S, Bailes J, Mccrea M, Harding H, Matthews a et al. Recurrent Concussion and Risk of Depression in Retired Professional Football Players. Medicine \& Science in Sports \& Exercise. 2007;39(6):903-9.

[21] Gandy S, Ikonomovic MD, Mitsis E, Elder G, Ahlers ST, Barth J, Stone JR, Dekosky ST, Chronic traumatic encephalopathy: Clinical-biomarker correlations and current concepts in pathogenesis. Mol Neurodegener. 2014;9(1):37.
[22] Guskiewicz K, Marshall S, Bailes J, McCrea M, Cantu $\mathrm{R}$, Randolph $\mathrm{C}$ et al. Association between Recurrent Concussion and Late-Life Cognitive Impairment in Retired Professional Football Players. Neurosurgery. 2005;719-26.

[23] Pickett T. Objectively assessing balance deficits after TBI: Role of computerized posturography. The Journal of Rehabilitation Research and Development. 2007;44(7):983-90.

[24] Walker W. Motor impairment after severe traumatic brain injury: A longitudinal multicenter study. The Journal of Rehabilitation Research and Development. 2007;44(7):97582.

[25] Kaufman K, Brey R, Chou L, Rabatin A, Brown A, Basford $\mathrm{J}$. Comparison of subjective and objective measurements of balance disorders following traumatic brain injury. Medical Engineering \& Physics. 2006;28(3):234-9.

[26] Geurts A, Ribbers G, Knoop J, van Limbeek J. Identification of static and dynamic postural instability following traumatic brain injury. Archives of Physical Medicine and Rehabilitation. 1996;77(7):639-44.

[27] Allison L. Imbalance following traumatic brain injury in adults. Neurology Report. 1999;23(1):13-18.

[28] Perez OH, Green RE, Mochizuki G. Characterization of balance control after moderate to severe traumatic brain injury: A longitudinal recovery study. Phys Ther, 2018;98(9):78695.

[29] Kelly J. Concussion in sports and recreation. Sem Neuro. 2000;20(02): 165-72.

[30] Hill J, Mobo B, Cullen M. Separating deployment-related traumatic brain injury and posttraumatic stress disorder in veterans. Am J of Phys Med \& Rehab. 2009;88(8):605-14.

[31] U.S. Army Medicine's Neurocognitive Assessment Branch. Rehabilitation \& Reintegration Division (2012). Automated Neuropsychological Assessment Metrics (ANAM) [brochure]. Retrieved from website: www.amedd.army. $\mathrm{mil} / \mathrm{r} 2 \mathrm{~d} / \mathrm{anam} . \mathrm{html} .2017$.

[32] Norris J, Carr W, Herzig T, Labrie D, Sams R. ANAM4 TBI reaction time-based tests have prognostic utility for acute concussion. Military Medicine. 2013;178(7):767-74.

[33] Brown C, Guskiewicz, K, Bleiberg J. Athlete characteristics and outcome scores for computerized neuropsychological assessment: A preliminary analysis. Journal of Athletic Training. 2017;42(4):515-23.

[34] Cernich A, Reeves D, Sun W, Bleiberg J. Automated Neuropsychological Assessment Metrics sports medicine battery. Archives of Clinical Neuropsychology. 2007;22:101-14.

[35] Girard P. Military and VA telemedicine systems for patients with traumatic brain injury. The Journal of Rehabilitation Research and Development. 2007;44(7):1017-26.

[36] Roebuck-Spencer T, Vincent A, Twillie D, Logan B, Lopez M, Friedl K et al. Cognitive change associated with self-reported mild traumatic brain injury sustained during the OEF/OIF conflicts. The Clinical Neuropsychologist. 2012;26(3):473-89.

[37] Acocello S, Broshek D, Saliba S. Comparison of brain acoustic monitor outcomes between healthy and concussed athletes 48 to 72 hours post-injury. Medicine \& Science in Sports \& Exercise. 2014;46:165.

[38] Dutton R, Sewell J, Aarabi B, Scalea T. Preliminary trial of a noninvasive brain acoustic monitor in trauma patients with severe closed head injury. The Journal of Trauma: Injury, Infection, and Critical Care. 2002;53(5):857-63.

[39] Dutton RP, Van Der Heijden MS, Aarabi B, Sewell J, Scalea TM (2005) Screening TBI patients with the brain acoustic 
monitor: Association with CT scan findings and neurologic status at hospital discharge, Clinical Intensive Care, 16:2, 97-105, DOI: 10.3109/09563070500116911.

[40] Jaeger M, Fröhlich M, Hertrich I, Ackermann H, Schönle P. Dysphonia subsequent to severe traumatic brain injury: Comparative perceptual, acoustic and electroglottographic analyses. Folia Phoniatrica et Logopaedica. 2001;53(6):326-37.

[41] McHenry M. Acoustic characteristics of voice after severe traumatic brain injury. The Laryngoscope. 2000;110(7):1157-61.

[42] Horner, T. Clinical Trials. Speech analysis as a diagnostic tool for mtbi [Internet]. 2014. Available from: http://http//clinicaltrials.gov/show/NCT0269795.

[43] Gamboa J, Jiménez-Jiménez F, Nieto A, Montojo J, OrtíPareja M, Molina $\mathrm{J}$ et al. Acoustic voice analysis in patients with Parkinson's disease treated with dopaminergic drugs. Journal of Voice. 1997;11(3):314-20.

[44] Garcia, M, Coreta, I., Martin, G., Alonso-Navarro, H., \& Jimenez-Jimenez F.J. Acoustic analysis of voice in Huntington's disease patients. Journal of Voice. 2011; 25(2):208-217.

[45] Mattson J. Secret Sounds ultimate healing. Oil City, PA; 2011.

[46] Sound Health [Internet]. Sound Health. 2019 [cited 27 February 2019]. Available from: https://www.soundheal thoptions.com/.

[47] Kay T, Harrington D, Adams R, Anderson T, Berrol $\mathrm{S}$, Cicerone $\mathrm{K}$ et al. Symptoms complaint following combat-related traumatic brain injury: Relationship to traumatic brain injury severity and posttraumatic stress disorder. Scandinavian Journal of Rehabilitation Medicine. 1993;23:179-85.

[48] Bleiberg J, Kane R, Reeves D, Garmoe W, Halpern E. Factor Analysis of Computerized and Traditional Tests Used in Mild Brain Injury Research. The Clinical Neuropsychologist (Neuropsychology, Development and Cognition: Section D). 2000;14(3):287-94.

[49] Johnson D, Vincent A, Johnson A, Gilliland K, Schlegel R. Reliability and construct validity of the Automated Neuropsychological Assessment Metrics (ANAM) mood scale. Archives of Clinical Neuropsychology. 2008;23(1): 73-85.

[50] Rice V, Lindsay G, Overby C, Jeter A, Boykin G, Alfred $\mathrm{P}$ et al. Human factors feedback: Brain acoustic monitor (TN469). Army Research Laboratory; 2012.

[51] Sound Recorder Software Free Download and Reviews [Internet]. Sound-Recorder.com. 2019 [cited 27 February 2019]. Available from: https://www.sound-recorder.com/

[52] Edwards, S. Vocal profiling for the professional: Illustrated reference guide. Available from http://www.sound healthinc.com. 2017.

[53] Concato J. The risk of determining risk with multivariable models. Annals of Internal Medicine. 1993;118(3):201.

[54] MacGregor A, Shaffer R, Dougherty A, Galarneau M, Raman R, Baker D et al. Prevalence and psychological correlates of traumatic brain injury in operation Iraqi freedom. Journal of Head Trauma Rehabilitation. 2010;25(1): $1-8$.

[55] Fagan T. Letter: Nomogram for Bayes theorem. The New England Journal of Medicine. 1975;293(5):857-63.

[56] Winter D. Human balance and posture control during standing and walking. Gait \& Posture. 1995;3(4):193-214.

[57] Matsuda S, Demura S, Demura T. Examining differences between center of pressure sway in one-legged and two-legged stances for soccer players and typical adults. Perceptual and Motor Skills. 2010;110(3):751-60.

[58] Raymond F. The ability to voluntarily control sway reflects the difficulty of the standing task. Gait \& Posture. 2010;31(1):78-81.

[59] Cornilleau-Pérès V, Shabana N, Droulez J, Goh J, Lee G, Chew P. Measurement of the visual contribution to postural steadiness from the COP movement: Methodology and reliability. Gait \& Posture. 2005;22(2):96-106.

[60] Houdijk H, Fickert R, van Velzen J, van Bennekom C. The energy cost for balance control during upright standing. Gait \& Posture. 2009;30(2):150-4.

[61] Doyle T, Newton R, Burnett A. Reliability of traditional and fractal dimension measures of quiet stance center of pressure in young, healthy people. Archives of Physical Medicine and Rehabilitation. 2005;86(10):2034-40.

[62] Findling O, Schuster C, Sellner J, Ettlin T, Allum J. Trunk sway in patients with and without, mild traumatic brain injury after whiplash injury. Gait \& Posture. 2011;34(4):473-8.

[63] Slobounov S, Cao C, Sebastianelli W, Slobounov E, Newell K. Residual deficits from concussion as revealed by virtual time-to-contact measures of postural stability. Clinical Neurophysiology. 2008;119(2):281-9.

[64] Johansson B, Berglund P, Rönnbäck L. Mental fatigue and impaired information processing after mild and moderate traumatic brain injury. Brain Injury. 2009;23(13-14):102740.

[65] Oliver J, Ponsford J, Curran C. Outcome following traumatic brain injury: A comparison between 2 and 5 years after injury. Brain Injury. 1996;10(11):841-8.

[66] Ziino C. Ponsford J. Measurement and prediction of subjective fatigue following traumatic brain injury. Journal of the International Neuropsychological Society. 2005;11(04):416-25.

[67] Ziino C, Ponsford J. Selective attention deficits and subjective fatigue following traumatic brain injury. Neuropsychology. 2006;20(3):383-90.

[68] Cantor J, Ashman T, Gordon W, Ginsberg A, Engmann C, Egan $\mathrm{M}$ et al. Fatigue after traumatic brain injury and its impact on participation and quality of life. Journal of Head Trauma Rehabilitation. 2008;23(1):41-51.

[69] Brooks N, McKinlay W, Symington C, Beattie A, Campsie L. Return to work within the first seven years of severe head injury. Brain Injury. 1987;1(1):5-19.

[70] Jorge R, Robinson R, Moser D, Tateno A, Crespo-Facorro B, Arndt S. Major depression following traumatic brain injury. Archives of General Psychiatry. 2004;61(1):42.

[71] McCleary C, Satz P, Forney D, Light R, Zaucha K, Asarnnow $\mathrm{R}$ et al. Depression after traumatic brain injury as a function of Glasgow outcome score. Journal of Clinical and Experimental Neuropsychology. 2017;270-9.

[72] Mooney G, Speed J. The association between mild traumatic brain injury and psychiatric conditions. Brain Injury. 2001;15(10):865-77.

[73] Silver J, Yudofsky S, Hales R. Depression in traumatic brain injury. Neuropsychiatry, Neuropsychology, \& Behavioral Neurology. 1991;4(1):12-13.

[74] Castriotta R, Wilde M, Lai J, Ananasov S, Masel B, Kuna $\mathrm{S}$. Prevalence and consequences of sleep disorders in traumatic brain injury. Journal of Clinical Sleep Medicine. 2007;3(4):349-56.

[75] Orff H, Ayalon L, Drummond S. Traumatic brain injury and sleep disturbance. Journal of Head Trauma Rehabilitation. 2009;24(3):155-65. 
[76] DePalma R, Burris D, Champion H, Hodgson M. Blast injuries. The New England Journal of Medicine. 2005;352(13):1335-42.

[77] Active Signal Technologies BAM (Brain Acoustic Monitor) [Internet]. Activesignaltech.com. 2019 [cited 27 February 2019]. Available from: http://activesignaltech. com/BAM1.html.

[78] Theodoros DG, Murdoch BE. Differential patterns of hyperfunctional laryngeal impairment in dysarthric speakers following severe closed head injury. In: Robin DA, Yorkston KM, Beukelman DR, eds. Disorders of Motor Speech: Assessment, Treatment, and Clinical Characterization. Baltimore: Paul H. Brookes, 205-227 1996.

[79] McHenry MA. Laryngeal airway resistance following traumatic brain injury. In: Robin DA, Yorkston KM, Beukelman DR, eds. Disorders of Motor Speech: Assessment, Treatment, and Clinical Characterization. Baltimore: Paul H. Brookes, 1996:229-40.

[80] McHenry M. Acoustic characteristics of voice after severe traumatic brain injury. The Laryngoscope. 2000;110:11571161. 110

[81] Daudet L, Yadav N, Perez M, Poellabauer C, Schneider, Huebner A. Portable mTBI assessment using temporal and frequency analysis of speech. IEEE Journal of Biomedical and Health Informatics. 2016;21(2):496-506.

[82] Jackson G. Detecting traumatic brain injury in the active duty U.S. Army. Neurorehabilitation. 2009;26(1):43-7.

[83] Hoge C, McGurk D, Thomas J, Cox A, Engel C, Castro C. Mild traumatic brain injury in U.S. soldiers returning from
Iraq. New England Journal of Medicine. 2008;358(5):45363.

[84] Kleffelgaard I, Roe C, Soberg H, Bergland A. Associations among self-reported balance problems, post-concussion symptoms and performance-based tests: A longitudinal follow-up study. Disability and Rehabilitation. 2011;34(9):788-94.

[85] Jaeschke R, Guyatt GH, Sackett DL. User's guides to the medical literature III. How to use an article about a diagnostic test. B. What are the results and will they help me in caring for my patients? JAMA 271(9), March 2, 1994.

[86] Wright WG, Tierney RT, McDevitt J. Visual-vestibular processing deficits in mild traumatic brain injury.

[87] Hoffer ME, Balaban C, Szczupak M, Buskirk J, Snapp H, Crawford J, Wise S, Murphy S Marshall K, Pelusso C, Knowles S, Kiderman A. The use of oculomotor, vestibular, and reaction time tests to assess mild traumatic brain injury (mTBI) over time. Laryngoscope Investigative Otolaryngology. 2017;2:157-65.

[88] Matheson L. Executive dysfunction, severity of traumatic brain injury, and IQ in workers with disabilities. Work. 2010;36(4), 413-22.

[89] McGinn T, Gordon H, Guyatt G, Wyer P, Naylor D, Stiell I et al. User's guide to the medical literature, XXII: How to use articles about clinical decision rules. The Journal of the American Medical Association. 2000;284(1):79-84 